\title{
Application of vascular endothelial growth factor at different phases of intestinal ischemia/reperfusion: What are its effects on oxidative stress, inflammation and telomerase activity?
}

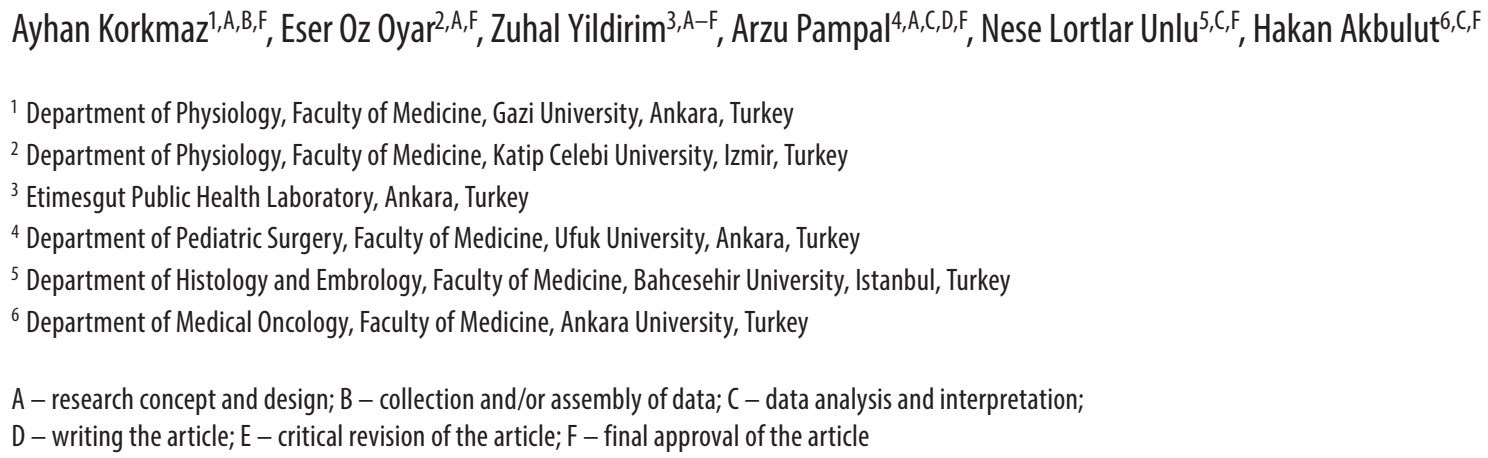

Address for correspondence

Zuhal Yildirim

E-mail: zyildirim2004@yahoo.com

Funding sources

None declared

Conflict of interest

None declared

Received on March 29, 2020

Reviewed on April 20, 2020

Accepted on August 11, 2020

Cite as

Korkmaz A, Oyar EO, Yildirim Z, Pampal A, Unlu NL, Akbulut H. Application of vascular endothelial growth factor at different phases of intestinal ischemia-reperfusion: What are its effects on oxidative stress, inflammation and telomerase activity? Adv Clin Exp Med. 2020:29(12):1417-1424. doi:10.17219/acem/126297

DOI

10.17219/acem/126297

Copyright

Copyright by Author(s)

This is an article distributed under the terms of the

Creative Commons Attribution 3.0 Unported (CC BY 3.0)

(https://creativecommons.org/licenses/by/3.0/)

\section{Abstract}

Background. Intestinal ischemic reperfusion injury (IRI) represents a great challenge in clinical practice, with high morbidity and mortality. Vascular endothelial growth factor (VEGF), as a signal protein, contributes to vasculogenesis and angiogenesis.

Objectives. To evaluate the local effectiveness of VEGF following intestinal IRI and its relation with application time.

Material and methods. Thirty Wistar albino rats were allocated to 5 groups and underwent laparotomy. The superior mesenteric arteries (SMA) were dissected in 4 groups, while the control group $(\mathrm{GrC})$ underwent a resection of small and large intestines. The VEGF group (Gr V) received VEGF following SMA dissection, with no further intervention, and the remaining 3 groups were subjected to ischemia for 90 min through occlusion of SMA and reperfusion for $4 \mathrm{~h}$. Ischemic reperfusion group (Gr I/R) received no additional medication, while the remaining 2 groups received VEGF just before ischemia ( $G r V+l / R)$ and during reperfusion $(G r l / R+V)$.

Results. Both applications of VEGF caused decreases in plasma levels of interleukin 6 (IL-6), tumor necrosis factor a (TNF-a), intestinal malondialdehyde (MDA), oxidized glutathione, protein carbonyl levels, and increases in intestinal total glutathione and superoxide dismutase (SOD) levels. Telomerase activity, which disappeared for $\mathrm{Gr} I / R$, was found to be elevated following both treatment groups. Similarly, the histopathological scores were found better for both treatment groups, but Gr V-I/R represented best outcomes.

Conclusions. The findings of our study revealed that VEGF, applied either before ischemia or during reperfusion, is effective on local damage following intestinal IRI. By interpreting the biochemical analysis and histopathological findings, we conclude either treatment option to be considered according to the reason of intestinal IRI.

Key words: inflammation, VEGF, telomerase activity, intestinal ischemic reperfusion injury, oxidative stress 


\section{Introduction}

Intestinal ischemic reperfusion injury (IRI), due to the occlusion at the level of superior mesentery artery (SMA), is connected to parenchymal damage at the intestine as a result of hypoxia, resultant adenosine triphosphate (ATP) depletion and intracellular acidosis at the early phase. The intestinal mucosa and submucosa show deterioration resulting from ischemic damage and ischemia, which also causes stagnation in the bowel and damage to the intestinal barriers. If the condition is untreated, the initial damage is exacerbated and ischemia of the muscle layers becomes inevitable, possibly leading to intestinal perforation, sepsis and even death. ${ }^{1}$ As the clinical problem is resolved in IRI, the main problem at the tissue level is the additional inflammatory process taking place due to reperfusion. ${ }^{2,3}$ Evidence supports local and systemic effects of IRI, as many injurious substances and factors are activated during IRI and are released to systemic circulation through venous and/or lymphatic routes. ${ }^{2-5}$ Moreover, IRI has been shown to induce apoptosis of not only intestinal cells but also remote organs as a result of reactive oxygen species (ROS) and pro-inflammatory cytokine production. ${ }^{4}$

Vascular endothelial growth factor (VEGF) is a participant of vital signaling proteins. As a mitogen agent for endothelium, VEGF induces vascular growth and increases microvascular permeability. It acts via nitric oxide (NO) dependent pathways and stimulates vasodilation. ${ }^{5}$ Vessel growth is induced by means of pre-existing vasculature and this expansion is important, especially in case of inadequate blood supply. Hypoxia contributes to angiogenesis, as it is the major stimulant producing VEGF and its receptors. ${ }^{6}$

The VEGF was demonstrated as a recuperative agent of IRI of various organs like myocardium, liver and spinal cord. ${ }^{7-11}$ Thus, we wanted to establish whether it is as effective on intestinal IRI as other organs - and if so, does the timing of its application matter. We designed a study in order to evaluate the effectiveness of VEGF on intestinal IRI used either before ischemia or during reperfusion.

\section{Material and methods}

The permission for the experimental study was granted by Animal Ethics Committee of Gazi University (Ankara, Turkey) and the study was completed due to the dictates of the Research Committee at the Experimental Research Center of Gazi University School of Medicine.

Thirty male adult Wistar albino rats weighing 220$270 \mathrm{~g}$ (mean weight $240 \pm 10 \mathrm{~g}$ ) were included in the study. All animals were housed under standard temperature and humidity with light and dark cycles of $10 \mathrm{~h}$ and $14 \mathrm{~h}$, respectively. No water, food and light restrictions were applied to animals. During the experiments, humane care was given to all animals according to Principles of Laboratory Animal Care and the Guide for the Care and Use of Laboratory Animals of National Health Institutes (NHS; Bethesda, USA). The animals were anaesthetized using xylazine hydrochloride ( $5 \mathrm{mg} / \mathrm{kg}$ ) (Alfazyne; Ege Vet, Izmir, Turkey) and ketamine hydrochloride (40 mg/kg) (Ketalar; Eczacibasi, Istanbul, Turkey), and every surgical procedure was performed under sterile conditions by the surgeon of the team (AP).

All the rats were randomly allocated to 5 groups and underwent a median laparotomy:

- Control group (Gr C, n = 6) underwent excision of small and large intestines with no further intervention.

- VEGF group (Gr V, n =6) underwent only dissection of SMA and received VEGF $(0.8 \mu \mathrm{g} / \mathrm{kg})$ through the caudal caval vein.

- IRI group (Gr I/R, n =6) underwent dissection and occlusion of SMA. After the dissection of SMA, the vessel was occluded for 90 min using a microclamp at the point where it branches from abdominal aorta. Pulseless mesenteric artery accompanying pale jejunoileal segments confirmed the ischemia.

- VEGF-IRI group (Gr V-I/R, $\mathrm{n}=6$ ) received VEGF $(0.8 \mu \mathrm{g} / \mathrm{kg})$ through caudal caval vein before the surgical procedure. The animals in this group underwent dissection, and occlusion of SMA for 90 min following drug administration.

- IRI-VEGF group (Gr I/R-V, n = 6) underwent dissection, and occlusion of SMA for $90 \mathrm{~min}$. The animals in this group received VEGF $(0.8 \mu \mathrm{g} / \mathrm{kg})$ through the caudal caval vein at the beginning of reperfusion.

The infusion dose for VEGF was chosen as the minimum effective dose as defined according to the previous study by Oz Oyar et al. ${ }^{7}$ Animals in all groups but $\mathrm{Gr} \mathrm{C}$ underwent a relaparotomy at the $4^{\text {th }}$ hour of reperfusion, and small bowel and proximal large bowel segments of the animals were removed. The rats were sacrificed with aspiration of cardiac blood and the blood samples were placed in EDTA tubes. Plasma samples were separated by centrifugation $\left(3000 \times \mathrm{g}\right.$ for $10 \mathrm{~min}$ at $\left.4^{\circ} \mathrm{C}\right)$ and frozen at $-80^{\circ} \mathrm{C}$. The excised bowel segment was washed in cold $0.9 \% \mathrm{NaCl}$, wiped, and weighed. To perform biochemical analysis and histopathological evaluation, the bowel was cut into 3 equal pieces. The $1^{\text {st }}$ (representing the most proximal portion) and $3^{\text {rd }}$ (representing the most distal portion) pieces were fixed in liquid nitrogen and kept frozen at $-80^{\circ} \mathrm{C}$, whereas the $2^{\text {nd }}$ (representing the middle portion) piece was laid in $10 \%$ neutral buffered formalin.

\section{Biochemical analysis}

\section{Western blot analysis}

Intestinal tissues were weighed, diluted with lysis buffer and homogenized at a ratio of $1 / 10(\mathrm{w} / \mathrm{v})$. Homogenates were centrifuged at $2500 \mathrm{rpm}$ for $10 \mathrm{~min}$ at $+4^{\circ} \mathrm{C}$. To quantify the protein levels, BCA Assay Kit (Pierce, Waltham, USA) was used. The heat shock protein (HSP70) (Rabbit, 
SPA810; Stressgen, San Diego, USA) and catalase (CAT) (Rabbit, ab1877; Abcam, Cambridge, UK) primary antibodies, used for western blotting analysis and $\beta$-actin, were used as housekeeping proteins in order to quantify the other primary antibodies.

\section{Determination of intestinal malondialdehyde levels}

Lipid peroxidation was measured using the test published by Gérard-Monnier et al. ${ }^{12}$ The malondialdehyde (MDA) levels were also recalculated from our MDA standards produced by the acid hydrolysis of 1,1,3,3-tetramethoxypropane.

\section{Determination of intestinal superoxide dismutase activity}

Superoxide dismutase (SOD) activity was measured using nitroblue tetrazolium (NBT) reduction in order to inhibit SOD activity. A superoxide generator (xanthine and xanthine oxidase) was used to trigger inhibition and the amount of SOD required to produce $50 \%$ inhibition was accepted to define 1 IU. ${ }^{13}$

\section{Determination of intestinal protein carbonyl group levels}

Protein carbonyl group (PC) levels were measured and described by Buss et al. ${ }^{14}$ Carbonyl residues were determined using 2,4-dinitrophenylhydrazine (DNPH) and carbonyl contents were calculated by obtaining the enzymelinked immunosorbent assay (ELISA) of the DNPH-treated samples at $480 \mathrm{~nm}$.

\section{Determination of intestinal total and oxidized glutathione levels}

Total glutathione (TGSH) and oxidized glutathione (GSSG) levels were determined with glutathione reductase (GR) as described by Tietze. ${ }^{15}$

\section{Determination of intestinal telomerase activity}

Telo TAGGG Telomerase PCR ELISA Plus kit (Roche, Mannheim, Germany) was used to determine the telomerase activity. Reactive telomerase activity was quantified with photometric enzyme immunoassay using TRAP and presented as percentage.

\section{Determination of intestinal hypoxia inducible factor 1a levels}

Hypoxia-inducible factor $1 \alpha$ (HIF- $1 \alpha)$ levels were defined with a solid phase sandwich ELISA (Rat HIF-1 $\alpha$ ELISA Kit; USCN Life Science Inc Wuhan, China; Cat No. E90798Ra, Lot No. 111011285).

\section{Plasma tumor necrosis factor a} and interleukin 6 measurements

Plasma tumor necrosis factor $\alpha$ (TNF- $\alpha$ ) and interleukin 6 (IL-6) levels were also determined by a solid phase sandwich ELISA (Rat TNF- $\alpha$ ELISA Kit; Cat No. ER3TNFA, Lot No. LF144120, Rat IL-6 ELISA Kit; Cat No. EH2IL-6, Lot No. KL138035; Thermo Fisher Scientific, Waltham, USA).

\section{Histopathological evaluation}

The intestinal pieces laid in $10 \%$ buffered formalin were fixed for $72 \mathrm{~h}$ and set in paraffin blocks. Afterwards, fivemicrometer-thick sections were cut axially using a microtome (RM 2245; Leica, Wetzlar, Germany). Hematoxylin and eosin (H\&E) staining was performed for the sections and the slides were evaluated under a light microscope (DMI 4000 B; Leica) by the histologist of the team (NLU) who was blind to the study groups. In order to quantify the ischemic changes at the mucosal level, a scoring system described by Chiu et al. was used. ${ }^{16}$

\section{Immunohistochemical evaluation}

After fixing in formalin, embedding in paraffin and cutting sections $5 \mu \mathrm{m}$ in thickness, tissue sections were placed in an incubator at $37^{\circ} \mathrm{C}$ for a night and at $60^{\circ} \mathrm{C}$ for $1 \mathrm{~h}$. Xylol was applied twice for $15 \mathrm{~min}$. The slides were then placed in $96 \%$ absolute alcohol, $80 \%$ ethanol for $10 \mathrm{~min}$ and distilled water twice for $5 \mathrm{~min}$. For caspase- $3 \mathrm{immu}-$ noperoxidase, the slides were placed in a high temperature microwave oven in $10 \%$ citrate buffer. After the tissue was left for $20 \mathrm{~min}$ at room temperature, it was marked with a hydrophobic pen (Super PAP PEN IM3580; Immunotech, Beckman Coulter Co., Brea, USA). In order to block endogenous peroxidase activity, the tissue was washed with distilled water and 0.02 M PBS (pH 7.2) (Lab Vision Corp., Fremont, USA) and $0.3 \%$ hydrogen peroxide (Thermo Fisher Scientific) was applied thereafter for $10 \mathrm{~min}$. After washing with PBS, ultra V block (TA-125-UB; Thermo Fisher Scientific) was utilized. Primary antibody including caspase-3 (rabbit polyclonal antibody; Lab Vision/ Neo Markers Corp.) was applied for $1 \mathrm{~h}$, the samples were washed with phosphate-buffered saline (PBS), and a postPBS level was applied (biotinylated goat anti-polyvalent and streptavidin peroxidase, respectively). After having been rewashed with $\mathrm{PBS}$, the specimens were placed for $10 \mathrm{~min}$ in AEC (3-amino, 9-ethylcarbazole) (TA_125-HA; Thermo Fisher Scientific) chromogen. Finally, the counterstain with Mayer hematoxylin was performed for $2 \mathrm{~min}$. All the slides were evaluated using a light microscope (DMI 4000 b; Leica) by the same histologist (NLU). The relative intensity of immunoreactivity staining was quantified as described by McCarty et al., focusing on the intensity and the distribution of a specific stain. ${ }^{17}$ A histological score (H-score) was formulated as the sum of the percentages 
of positively stained cells multiplied by the weighted intensity of staining. The $\mathrm{H}$-score was calculated with the formulation of $\sum \mathrm{Pi}(\mathrm{I}+1)$, as I represented intensity of staining ( 0 - none; 1 - mild; 2 - moderate; and 3 - intense) and Pi represented the fraction of stained cells for each intensity.

\section{Statistical analysis}

Statistical package SPSS for Windows v. 18.0 (SPSS Inc., Chicago, USA) was used for analyses with the descriptive statics presented as mean \pm standard deviation (SD). Kruskal-Wallis test and Mann-Whitney U test were used for statistical analyses and Spearman's correlation test was used to evaluate the correlation between variables. A p-value less than 0.05 was considered to be statistically significant.

\section{Results}

All the animals survived the experiment. The results obtained from each group are presented in Tables 1, 2.

\section{Intestinal MDA levels}

The MDA levels for proximal and distal intestinal segments of $\mathrm{Gr}$ I/R were elevated when compared to $\mathrm{Gr} \mathrm{C}$ $(\mathrm{p}<0.05)$. All treatment groups, not being superior to one another, represented a significant decrease of MDA level at the proximal segment whereas only Gr V-I/R represented a significant decrease at the distal segment (Table 1).

Table 1. Biochemical analysis of intestinal segments and plasma

\begin{tabular}{|c|c|c|c|c|c|}
\hline \multirow{2}{*}{ Parameter } & \multicolumn{5}{|c|}{ Group } \\
\hline & Control & VEGF & $\mathrm{I} / \mathrm{R}$ & V-I/R & I/R-V \\
\hline MDA-proximal $[\mu \mathrm{M}]$ & $4.08 \pm 0.40$ & $4 \pm 0.18$ & $8.16 \pm 1.56^{*}$ & $4.87 \pm 0.58^{\#}$ & $4.99 \pm 0.68^{\#}$ \\
\hline MDA-distal $[\mu \mathrm{M}]$ & $3.29 \pm 0.46$ & $3.3 \pm 0.71$ & $8.44 \pm 2.97^{*}$ & $4.84 \pm 1.19^{\#}$ & $6.84 \pm 1.51$ \\
\hline TGSH-proximal $[\mu \mathrm{M}]$ & $326 \pm 32.78$ & $317.2 \pm 13.6$ & $183.16 \pm 31.5^{*}$ & $299.66 \pm 86.48^{\#}$ & $250.33 \pm 48.74$ \\
\hline TGSH-distal [ $\mu \mathrm{M}]$ & $488.45 \pm 48.76$ & $467.26 \pm 60.55$ & $160.63 \pm 35.77^{*}$ & $341 \pm 42.78^{\#}$ & $290.32 \pm 60.9^{\#}$ \\
\hline GSSG-proximal [ $\mu \mathrm{M}]$ & $3.93 \pm 1.39$ & $3.91 \pm 0.76$ & $8.29 \pm 1.46^{*}$ & $3.62 \pm 1.19^{\#}$ & $4.66 \pm 0.83^{\#}$ \\
\hline GSSG-distal [ $\mu \mathrm{M}]$ & $5.28 \pm 1.36$ & $5.5 \pm 1.37$ & $14.03 \pm 2.19^{*}$ & $6.07 \pm 0.97^{\#}$ & $6.58 \pm 1.01^{\#}$ \\
\hline SOD-proximal [AU] & $6.15 \pm 1.32$ & $6.9 \pm 0.31$ & $3.32 \pm 0.75^{*}$ & $5.92 \pm 0.89$ & $5.33 \pm 1.71$ \\
\hline SOD-distal [AU] & $8.54 \pm 2.07$ & $8.65 \pm 1.52$ & $3.54 \pm 0.94^{*}$ & $7.02 \pm 2.28^{\#}$ & $5.35 \pm 1.31^{\#}$ \\
\hline PC-proximal [AU] & $0.103 \pm 0.013$ & $0.101 \pm 0.005$ & $0.114 \pm 0.011$ & $0.097 \pm 0.010$ & $0.105 \pm 0.006$ \\
\hline PC-distal [AU] & $0.191 \pm 0.004$ & $0.194 \pm 0.002$ & $0.199 \pm 0.003^{*}$ & $0.172 \pm 0.027^{\#}$ & $0.184 \pm 0.008^{\#}$ \\
\hline CAT-proximal [AU] & $0.83 \pm 0.06$ & $0.84 \pm 0.16$ & $0.47 \pm 0.09^{*}$ & $0.55 \pm 0.1^{\#}$ & $0.67 \pm 0.15$ \\
\hline CAT-distal [AU] & $1.13 \pm 0.14$ & $1.18 \pm 0.13$ & $0.69 \pm 0.12$ & $0.79 \pm 0.04$ & $0.85 \pm 0.12$ \\
\hline HSP70-proximal [AU] & $0.51 \pm 0.24$ & $0.49 \pm 0.2$ & $0.82 \pm 0.18^{*}$ & $0.67 \pm 0.2$ & $0.64 \pm 0.39$ \\
\hline HSP70-distal [AU] & $0.74 \pm 0.13$ & $0.75 \pm 0.16$ & $1.06 \pm 0.55$ & $0.81 \pm 0.21$ & $0.79 \pm 0.17$ \\
\hline Telomerase activity [\%] & 100 & 83.33 & $0^{*}$ & $50^{\#}$ & $66.67^{\#}$ \\
\hline Intestinal HIF-1a [ng/mL] & $9.62 \pm 4.02$ & $13.89 \pm 2.45$ & $15.01 \pm 0.51^{*}$ & $13.84 \pm 2.02$ & $13.56 \pm 3.51$ \\
\hline Plasma TNF-a [pg/mL] & $124.22 \pm 20.70$ & $110.39 \pm 19.90$ & $326.27 \pm 84.93^{*}$ & $139.06 \pm 5.05^{\#}$ & $114.76 \pm 14.93^{\#}$ \\
\hline Plasma IL-6 [pg/mL] & $16.30 \pm 1.81$ & $29.78 \pm 5.41$ & $280.24 \pm 89.96^{*}$ & $131.69 \pm 24.87^{\#}$ & $73.65 \pm 6.67^{\#, \&}$ \\
\hline
\end{tabular}

Biochemical analysis of intestinal segments and plasma, results presented as mean \pm standard deviation (SD); ${ }^{*} p<0.05$ compared to control group; \# $\mathrm{p}<0.05$ compared to I/R; \& $\mathrm{p}<0.05$ when compared to V-I/R group; VEGF - vascular endothelial growth factor; MDA - malondialdehyde; TGSH - total glutathione; GSSG - oxidized glutathione; SOD - superoxide dismutase; PC - protein carbonyl group; CAT - catalase; HSP - housekeeping protein; HIF-1a - hypoxia-inducible factor 1a; TNF-a - tumor necrosis factor a; IL- 6 - interleukin 6.

Table 2. Mean histopathological scoring, intestinal caspase-3 immunoreactivity and MMP-9 levels for all groups

\begin{tabular}{|l|c|c|c|c|}
\multicolumn{1}{|c|}{ Parameter } & Control & VEGF & I/R & V-I/R \\
\hline $\begin{array}{l}\text { Mean histopathologic } \\
\text { scores (grade) }\end{array}$ & 0.16 & 0.33 & $2.66^{*}$ & $0.5^{\#, \&}$ \\
\hline Caspase-3 & $0.85 \pm 0.37$ & $1.07 \pm 0.41$ & $2.95 \pm 0.45^{*}$ & $1.02 \pm 0.45^{\#}$ \\
\hline MMP-9 & $1.78 \pm 0.35$ & $1.83 \pm 0.42$ & $3.07 \pm 0.20^{*}$ & $2.15 \pm 0.46^{\#}$ \\
\hline
\end{tabular}

Intestinal caspase-3 immunoreactivity and MMP-9 levels, results presented as mean \pm standard deviation (SD); ${ }^{*} \mathrm{p}<0.05$ compared to control group;

\# $\mathrm{p}<0.05$ when compared to I/R group; \& $\mathrm{p}<0.05$ compared to I/R-V group); VEGF - vascular endothelial growth factor; MMP-9 - matrix metalloproteinase 9. 


\section{Intestinal SOD activity}

The SOD activity of both proximal and distal intestinal segments of Gr I/R were found decreased when compared to $\mathrm{Gr} \mathrm{C}(\mathrm{p}<0.05)$. Either treatment represented a significant elevation only for the distal segment (Table 1).

\section{Intestinal PC levels}

The PC levels of distal intestinal segments of Gr I/R were found increased when compared to $\operatorname{Gr} C(\mathrm{p}<0.05)$ and either treatment represented significant fall for the same segment (Table 1).

\section{Intestinal CAT activity}

Catalase activity was found to decrease significantly at the proximal segment in $\mathrm{Gr} I / \mathrm{R}$ when compared to $\mathrm{Gr} C$ $(\mathrm{p}<0.05)$ and the rise of CAT was statistically significant only at the proximal segment for Gr V-I/R (Table 1).

\section{Intestinal TGSH and GSSG levels}

A statistically significant increase in TGSH level and a statistically significant decrease in GSSG level were seen at both proximal and distal intestinal segments of Gr I/R when compared to Gr C. Gr V-I/R represented a significant increase in TGSH for both segments, whereas Gr I/R-V represented a significant rise at the distal segment. Both treatment groups represented a significant decrease in GSSG for both segments, but neither treatment was found superior (Table 1).

\section{Intestinal telomerase activity}

Telomerase activity was found to decrease to 0 in Gr I/R when compared to $\mathrm{Gr} C(\mathrm{p}<0.05)$ and all treatment groups recuperated the telomerase activity significantly (Table 1 ).

\section{Intestinal HIF-1a levels}

The HIF-1 $\alpha$ levels were found increased in Gr I/R when compared to Gr C ( $<0.05)$, but the decrease in treatment groups were insignificant (Table 1$)$.

\section{Plasma TNF- $a$, and IL- 6 levels}

Both of the parameters were found significantly increased in Gr I/R when compared to Gr C. Either treatment group represented a significant decrease for TNF- $\alpha$, and IL-6 when compared to Gr I/R. Despite no changes in TNF- $\alpha$ levels, Gr I/R-V had a more significant decrease to $\mathrm{Gr} \mathrm{V}-\mathrm{I} / \mathrm{R}$ in terms of decrease in IL-6 levels $(\mathrm{p}<0.05)$ (Table 1).

\section{Histopathological evaluation}

Gr I/R represented significant ischemic changes, such as generalized desquamation and moderate to severe mucosal congestion, when compared to $\mathrm{Gr}$ C. Both treatment groups represented enhanced histopathological findings, such as focal desquamation and mild mucosal congestion (Fig. 1). The comparison of histopathological scoring of treatment groups revealed a better outcome for $\mathrm{Gr} \mathrm{V}-\mathrm{I} / \mathrm{R}$ when compared to $\mathrm{Gr} I / \mathrm{R}-\mathrm{V}$ with a statistically significant difference (Table 2). In terms of caspase activity and matrix metalloproteinase 9 (MMP-9) scores, Gr I/R represented the highest score and both treatment groups represented better scores when compared to Gr I/R $(\mathrm{p}<0.05)$ (Table 2).

\section{Discussion}

The VEGF is a key signal protein that stimulates expansion of new vasculature from surviving vessels in case of hypoxia. ${ }^{7}$ This angiogenic activity is also supported by the vasodilator effect of protein through nitric oxide (NO) production. The VEGF is also shown to have antioxidant effects and to modulate endothelial dysfunction during IRI. In this study, we aimed to evaluate the effectiveness of VEGF on intestinal IRI during the different phases of the injury. For this purpose, experimental intestinal IRI model was established as described by Dwivedi et al. and an intravenous VEGF dose of $0.8 \mu \mathrm{g} / \mathrm{kg}$ was used for treatment, as this dose was proven to be the minimal effective concentration in an earlier study. ${ }^{11,18}$ The VEGF was applied either before ischemia or during the reperfusion of the experimental intestinal IRI model and it was found that the drug was effective in both situations. This is also the first study that evaluated the changes of the bowel at separate levels (proximal and distal bowel segments) after ischemia and reperfusion. Both of the treatment groups represented enhancement in terms of tissue MDA, TGSH/GSSG, SOD, PC, and CAT levels for both intestinal segments. However, some of the parameters showed different distributions - for example, better results with distal intestinal segments and better results with VEGF treatment before intestinal I/R. The differences between proximal and distal intestinal segments can be attributed to the anatomic settlement. Marginal artery (of Drummond) is the major anastomosis between superior and inferior mesenteric arteries. The marginal artery forms a continuous arterial circle along the inner border of the colon and it is probable that during SMA occlusion and reperfusion period the distal segments of the intestine was supported by inferior mesenteric artery (IMA) and presented better results. We observed that some parameters were recuperated only for Gr V-I/R (like MDA for distal segments, TGSH and MDA for proximal segments). Application time of VEGF resulted in a difference that is of interest and difficult to justify. 


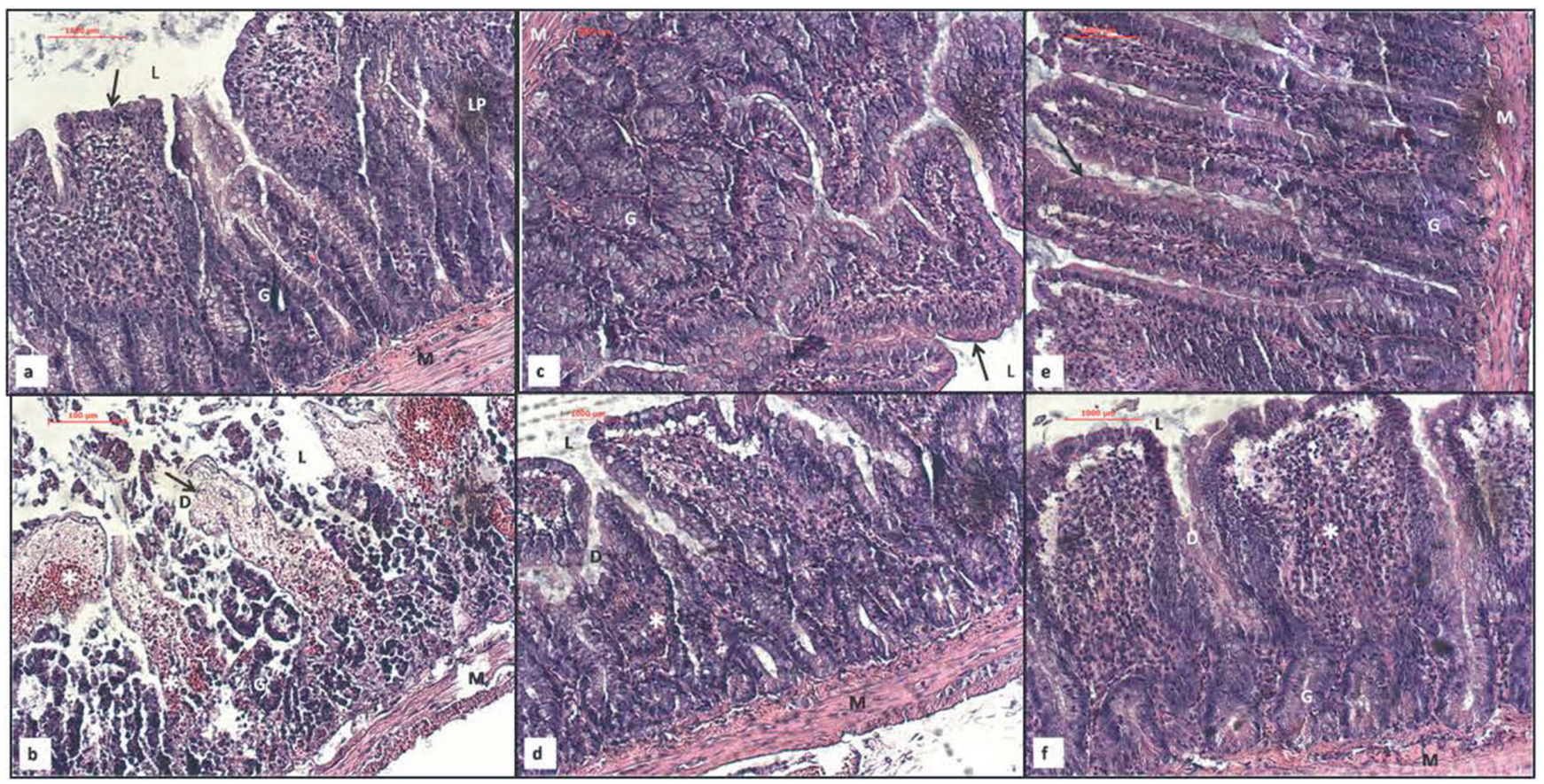

Fig. 1. Histopathological view of intestinal segments stained with H\&E in all groups. Panel (a) represents intestinal structures of Gr C within normal limits; (b) represents generalized desquamation (D) within intestinal epithelium and mucosal congestion (*) in Gr I/R; (c) represents intestinal structures of Gr V within normal limits; (d) represents localized desquamation (D) and mucosal congestion $\left(^{*}\right)$ with intact muscular layer in Gr l/R-V; (e) represents intestinal structures within normal limits of Gr V-I/R; and (f) represents localized desquamation (D) and mucosal congestion (*) of Gr V-I/R. Arrow - intestinal epithelium; L - intestinal lumen; G - intestinal secretory glands; LP - lamina propria; M - muscular layer; Bar $=1000 \mu$

Tissue hypoxia is inevitable as a result of capillary perfusion failure. Activation of hypoxia-inducible factor (HIF-1), which is the main regulator of pathophysiological response to hypoxia, has been shown to play an important role in inducing intestinal damage. This is why HIF- $1 \alpha$, the subunit of heterodimeric transcription factor HIF-1, is accepted as the main transcriptional regulator for cellular hypoxia. However, HIF-1 $\alpha$, constantly produced and kept low under normoxic conditions, increases rapidly during hypoxia and dimerizes with constitutively present HIF-1 $\beta$ to stimulate VEGF production. ${ }^{19,20}$ Studies evaluating the effects of IRI on HIF- $1 \alpha$ at separate organs revealed that the ischemic injury is related to elevation of HIF- $1 \alpha .^{21,22}$ Also, Poynter et al. represented the relation between HIF- $1 \alpha$ and VEGF in an experimental ischemic myocardial injury. ${ }^{21}$ The findings of our study supported HIF- $1 \alpha$ elevation in Gr I/R, but apart from the other studies, even the treatment groups represented a decrease in HIF-1 $\alpha$ levels; such decrease was statistically insignificant. As mentioned previously, HIF- $1 \alpha$ is increased rapidly during hypoxia and dimerized with HIF- $1 \beta$ in order to stimulate VEGF production and induce angiogenesis. Although a decrease in HIF-1 $\alpha$ levels is expected in the case of exogenous administration of VEGF, no such relationship was found in the findings of our study. It is probable that either HIF- $1 \alpha$ production is not affected by VEGF levels or the appearance of free radicals resulted in HIF- $1 \alpha$ being suspended in intestinal tissues despite its short half-life period.
Telomere is a DNA-protein complex consisting of 6 base repetitive sequences and telomere binding proteins at the end of the chromosomes of eukaryotic organisms. Telomeres protect against events such as disruption of the terminal regions of chromosomes. However, telomere segments are shortened by the cell cycle and cell division, which triggers cellular apoptosis. ${ }^{23}$ Telomerase is a ribonucleoprotein reverse transcriptase that adds telomeric DNA repeat sequences to the 3'-end of chromosomes. Despite its essential role for telomere homeostasis, its activation leads to unlimited cell proliferation, probably resulting in neoplastic transformation. However, this reverse transcriptase also reactivates during tissue repair. ${ }^{24}$ Eckers et al. reported that oxidative stress and elevated ROS are closely related to endothelial cell apoptosis and endothelial dysfunction, loss of telomerase activity and telomere shortening. ${ }^{25}$ Werner et al. presented the protective effect of upregulated telomerase activity against endothelial apoptosis in case of oxidative stress. ${ }^{26}$ Zaccagnini et al. showed that there was a positive regulation of VEGF and telomerase activity in a hind limb ischemia study in rats. ${ }^{27}$ The IRI related oxidative stress was also obtained in our study and we found that telomerase activity disappeared in the I/R group, and both treatment groups exhibited elevated telomerase activity, supporting reactivation of telomerase against injury.

The heat shock proteins are a class of molecular chaperons that exist in cell cytoplasm in order to prevent 
the formation of nonfunctional structures. It is thought to act as a cytosolic chaperone that plays a role in facilitating protein folding, degradation, complex assembly, and translocation. The best known members are the heat-inducible form, also known as HSP72 or HSP70i, and are also present in the structural form, also called HSC70, HSP73 or HSC73. ${ }^{28}$ HSP70 interacts with hydrophobic peptide parts of proteins that are not ATP-bound. These cytoprotective proteins are induced by many factors, such as infection, oxidative stress, osmotic stress, hyperthermia, ultraviolet exposure, and heavy metals. In the presence of these inducers, chaperone properties have been reported to reduce protein aggregates and intracellular inclusions. The catalytic unit of telomerase (TERT) is known to interact with chaperone proteins. The $70 \mathrm{kDa}$ heat shock proteins (HSP70), as a chaperone protein was shown not to be vital for telomerase activity but also to be important for telomerase stability. ${ }^{24}$ The literature review revealed a number of IRI studies on various organs representing elevation of HSP70. ${ }^{29}$ We also evaluated the HSP70 levels in our study and found the significant elevation of HSP70 at only the proximal intestinal segment, where IMA does not supply in $\mathrm{Gr} I / \mathrm{R}$, supporting the vascular compromise in that region. HSP70 was shown to be a cell-protector for apoptosis, but its overexpression was generally proven to be related to poor prognosis. ${ }^{30}$ Despite insignificant changes in HSP70 in our treatment groups, caspase activity was found to be elevated with IRI and the treatment with VEGF normalized this activity. Even though the last step of either intrinsic or extrinsic pathways of apoptosis is caspase-3 activation, DNA damage and heat shock proteins are mainly involved in intrinsic pathway. However, the increase of HSP70 and caspase-3 in Gr I/R is rational, but the presence of caspase activation in the treatment groups despite low HSP70 is beyond our predictions. These findings supported the claim that the extrinsic pathway stimulated abundantly during $I / R$ is somehow less affected.

Intestinal IRI is a clinical entity related to life-threatening conditions, such as mesenteric artery occlusion, intestinal transplantation, intussusceptions, acute blood loss, and trauma. Each of these conditions, however, causes the same physiological consequence and requires separate approaches in the clinical settings. In this study, we applied VEGF either before ischemia or during the reperfusion of the experimental intestinal IRI model and aimed to evaluate whether any changes occur. We found that the drug was effective in terms of enhancing intestinal IRI in both situations; apart from that, the treatment before ischemic process was found slightly superior to the other therapy. In the light of these findings, we can probably speculate on the benefits of VEGF usage of different timetables for different causes of intestinal IRI. It is obvious that further studies are needed to support this inference.

\section{Conclusions}

Intestinal IRI is a clinical entity related to life-threatening conditions like mesenteric artery occlusion, intestinal transplantation, intussusception, acute blood loss, and trauma. Each of these conditions, however, causes the same physiological consequence and has separate approaches in the clinical settings. That is why, the use of VEGF at different timetables of the ischemic reperfusion process could serve different outcomes for different reasons of IRI.

\section{ORCID iDs}

Ayhan Korkmaz (1) https://orcid.org/0000-0001-5702-8184 Eser Oz Oyar (10) https://orcid.org/0000-0003-1805-4149

Zuhal Yildirim (1) https://orcid.org/0000-0002-2808-7860

Arzu Pampal (10) https://orcid.org/0000-0002-7771-0380

Nese Lortlar Unlu (1) https://orcid.org/0000-0002-1716-9350

Hakan Akbulut (1) https://orcid.org/0000-0003-1631-5739

\section{References}

1. Schellekens D, Reisinger KW, Lenaerts K. SM22: A plasma biomarker for human transmural intestinal ischemia. Ann Surg. 2018;268(1): $120-126$.

2. Kesik V, Guven A, Vurucu S, et al. Melatonin and $1400 \mathrm{~W}$ ameliorate both intestinal and remote organ injury following mesenteric ischemia/reperfusion. J Surg Res. 2009;157(1):97-105.

3. Sagiroglu T, Sezer A, Altaner S, Umit H, Yalta T, Yagci MA. The effects of sirolimus on target organs during mesenteric ischemia and reperfusion damage in an experimental rat model. Curr Ther Res Clin Exp. 2011;72(2):79-93.

4. Al-Salam S, Hashmi S. Myocardial ischemia reperfusion injury apoptotic, inflammatory and oxidative stress role of galectin-3. Cell Physiol Biochem. 2018;50(3):1123-1139.

5. Oz Oyar E, Korkmaz A, Kardeş O, Ömeroğlu S. Aortic cross-clampinginduced spinal cord oxidative stress in rabbits: The role of a novel antioxidant adrenomedullin. J Surg Res. 2008;147(1):143-147.

6. Ferrara N. Role of vascular endothelial growth factor in regulation of physiological angiogenesis. Am J Physiol Cell Physiol. 2001;280(6): 1358-1366.

7. Oz Oyar E, Kardeş O, Korkmaz A, Ömeroğlu S. Effects of vascular endothelial growth factor on ischemic spinal cord injury caused by aortic cross-clamping in rabbits. J Surg Res. 2009;151(1):94-99.

8. Kirisci M, Oktar GL, Ozogul C, et al. Effects of adrenomedullin and vascular endothelial growth factor on ischemia/reperfusion injury in skeletal muscle in rats. J Surg Res. 2013;185(1):56-63.

9. Yang $Y$, Shi C, Hou X, et al. Modified VEGF targets the ischemic myocardium and promotes functional recovery after myocardial infarction. J Control Release. 2015;213:27-35.

10. Li GH, Luo B, Lv YX, et al. Dual effects of VEGF-B on activating cardiomyocytes and cardiac stem cells to protect the heart against shortand long-term ischemia-reperfusion injury. J Trans/ Med. 2016;14(1): 116-130.

11. Cao D, Wang M, Gong J, Wei S, Gong J, Li J. Exogenous vascular endothelial growth factor delivery prior to endothelial precursor cell transplantation in orthotopic liver transplant-induced hepatic ischemia reperfusion injury. Liver Transplant. 2017;23(6):804-812.

12. Gérard-Monnier D, Erdelmeier I, Régnard K, Moze-Henry N, Yadan JC, Chaudière J. Reactions of 1-methyl-2-phenylindole with malondialdehyde and 4-hydroxyalkenals: Analytical applications to a colorimetric assay of lipid peroxidation. Chem Res Toxicol. 1998;11(10):1176-1183.

13. Beauchamp C, Fridovich I. Superoxide dismutase: Improved assays and an assay applicable to acrylamide gels. Anal Biochem. 1971;44(1): 276-287.

14. Buss H, Chan TP, Shis KB, Domigan NM, Winterboum CC. Protein carbonyl measurement by a sensitive ELISA method. Free Radic Biol Med. 1997;23(3):361-366.

15. Tietze F. Enzymic method for quantitative determination of nanogram amounts of total and oxidized glutathione: Applications to mammalian blood and other tissues. Anal Biochem. 1969;27(3):502-522. 
16. Chiu CJ, McArdle AH, Brown R, Scott HJ, Gurd FN. Intestinal mucosal lesion in low-flow states. I. A morphological, hemodynamic, and metabolic reappraisal. Arch Surg. 1970;101:478-483.

17. McCarty KS Jr, Miller LS, Cox EB, Konrath J, McCarty KS Sr. Estrogen receptor analyses: Correlation of biochemical and immunohistochemical methods using monoclonal antireceptor antibodies. Arch Pathol Lab Med 1985;109(8):716-721.

18. Dwivedi AJ, Wu R, Nguyen E, et al. Adrenomedullin and adrenomedullin-binding protein-1 prevent acute lung injury after gut ischemia-reperfusion. J Am Coll Surg. 2007;205(2):284-293.

19. Hoeben A, Landuyt B, Highley MS, Wilders H, Van Oosterom AT, De Brujin EA. Vasculer endothelial growth factor and angiogenesis. Pharmacol Rev. 2004;56(4):549-580.

20. Mahfoudh-Boussaid A, Zaouali MA, Hadj-Ayed K, et al. Ischemic preconditioning reduces endoplasmic reticulum stress and upregulates hypoxia inducible factor-1a in ischemic kidney: The role of nitric oxide. J Biomed Sci. 2012;19(1):7-15.

21. Poynter JA, Manukyan MC, Wang $Y$, et al. Systemic pretreatment with dimethyloxalylglycine increases myocardial HIF-1a and VEGF production and improves functional recovery after acute ischemia/ reperfusion. Surgery. 2011;150(2):278-283.

22. Grenz A, Clambey E, Eltzschig HK. Hypoxia signaling during intestinal ischemia and inflammation. Curr Opin Crit Care. 2012;18(2):178-185.
23. Wyatt HD, West SC, Beattie TL. InTERTpreting telomerase structure and function. Nucleic Acids Res. 2010;38(17):5609-5622.

24. Pandita TK. Role of HSPs and telomerase in radiotherapy. Int J Hyperthermia. 2005;21(8):689-694.

25. Eckers A, Altschmied J, Haendeler J. Oxidative stress in endothelial cells and in diabetes type 2 [in German]. Z Geronto/ Geriatr. 2012;45(2): 90-94.

26. Werner C, Gensch C, Pöss J, Haendeler J, Böhm M, Laufs U. Pioglitazone activates aortic telomerase and prevents stress-induced endothelial apoptosis. Atherosclerosis. 2011;216(1):23-34.

27. Zaccagnini G, Gaetano C, Pietra LD, et al. Telomerase mediates vascular endothelial growth factor-dependent responsiveness in a rat model of hind limb ischemia. J Biol Chem. 2005;280(15):14790-14798.

28. Quyang YB, Giffard RG. MicroRNAs regulate the chaperone network incerebral ischemia. Trans/ Stroke Res. 2013;4(6):693-703.

29. Xiao B, Ma LL, Xiao CL, et al. Protective effect of heat shock protein 70 and magnesium sulfate supplementation on renal ischemia reperfusion injury [in Chinese]. Beijing Da Xue Xue Bao. 2011;43(4):525-530.

30. Kim BK, Kim BR, Lee HJ, et al. Tumor-suppressive effect of a telomerase-derived peptide by inhibiting hypoxia-induced HIF-1a-VEGF signaling axis. Biomaterials. 2014;35(9):2924-2933. 